\title{
Understanding neutrino cross sections for future oscillation experiments
}

\section{Artur M. Ankowski*}

Center for Neutrino Physics, Virginia Tech, Blacksburg, Virginia 24061, USA

E-mail: artankevt.edu

\begin{abstract}
The success of the next generation of neutrino-oscillation experiments requires significant reduction of systematic uncertainties. In this context, the description of nuclear effects in neutrino interactions is of particular importance, as it currently is one of the main systematic effects. Here I discuss the relevance of realistic description of the ground-state properties of the target nucleus, pointing out the importance of developing accurate theoretical models, capable to provide quantitative description of neutrino cross sections, together with the relevance of their implementation in Monte Carlo generators and extensive testing against lepton-scattering data.
\end{abstract}

Neutrino Oscillation Workshop

4 - 11 September, 2016

Otranto (Lecce, Italy)

\footnotetext{
*Speaker.
} 
Thanks to global efforts over the past two decades that yielded a number of experiments, neutrino oscillations are now well established. This phenomenon is being studied with an increasing precision, and within the next two decades, the uncertainties are expected to be sufficiently low to begin testing the unitarity of the mixing matrix, discover the mass hierarchy and unambiguously determine if neutrino mixing violates the charge-parity symmetry [1,2]. These results are going to have profound consequences for possible extensions of the Standard Model, building models of the neutrino masses, and our understanding of the matter-antimatter asymmetry in the Universe.

Owing to polychromatic neutrino beams employed, to extract the oscillation parameters from the collected event distribution, neutrino energies have to be reconstructed on an event-by-event basis from the measured kinematics of particles in the final state, and the accuracy of reconstruction translates into the accuracy of the determined oscillation parameters. Because of the low cross sections involved, it is necessary to employ nuclear targets as detector materials to ensure high statistics of collected events. As a consequence, however, description of nuclear effects turns out to be one of the largest sources of systematic uncertainties, playing an important role both in the energy reconstruction and the expected event-rate calculations. A significant reduction of these uncertainties is a prerequisite for the realization of the ambitious program of the next generation of neutrino measurements. A number of systematic uncertainties has been discussed in Ref. [3]. Here I will only analyze the relevance of realistic description of the ground-state properties of the target nucleus.

It is important to note that when the interaction process involves only one nucleon-with the remaining $(A-1)$ ones acting as spectators-neutrino and electron scattering are subject to the same nuclear effects. The nuclear cross section in this regime is a convolution of the elementary cross section - different for electrons and neutrinos—-with the hole and particle spectral functions (SFs) - common for electrons and neutrinos—describing the ground-state properties of the target and propagation of the struck nucleon, respectively [4]. As a consequence, models of nuclear effects in neutrino interactions can be validated by systematic comparisons to electron-scattering data at the kinematics of interest.

Even when more complex dynamics is involved, due to the similarity of the electromagnetic and weak interactions, it is highly improbable that a theoretical approach unable to reproduce electron-scattering data would be able to describe nuclear effects in neutrino scattering. Moreover, electron cross sections-double differential and for monoenergetic beams-allow for much easier understanding of discrepancies between theoretical results and experimental data than neutrino ones, in which different probe's energies and reaction mechanisms are intertwined.

As an illustrative example, the left panel of Fig. 1 compares the quasielastic (QE) cross sections obtained within the SF approach [5] and the relativistic Fermi gas (RFG) model [6] with the data [7]. Whereas the RFG results cannot reproduce heights, shapes and positions of the QE peaks, the SF calculations - although they do not involve any adjustable parameters - turn out to be in very good agreement with the experimental points.

Using the realistic ground-state description [8, 9], the SF calculations take into account both the shell structure and correlations between nucleons. The latter cause partial depletion of the shells-lowering the QE peaks—and give rise to high-momentum nucleons deeply bound in quasideuteron pairs-producing the tails at low values of the final electron energy $E_{e}^{\prime}$, which render the QE peaks asymmetric. In addition, the SF calculations [5] include the effect of final-state 

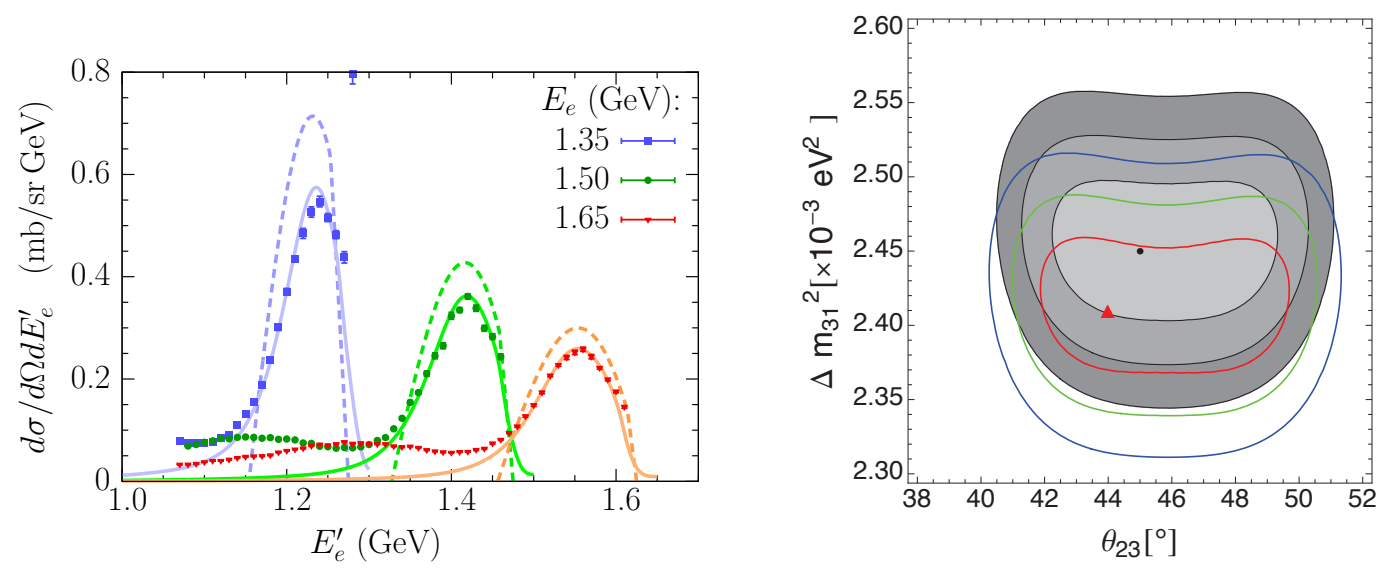

Figure 1: Left: Importance of an accurate description of nuclear effects illustrated by a comparison of the RFG (dashed lines) and SF [5] (solid lines) calculations of the double differential $\mathrm{C}\left(e, e^{\prime}\right)$ cross sections with the data for scattering angle $13.5^{\circ}$ and beam energies $1.35,1.50$ and $1.65 \mathrm{GeV}$ [7]. Right: Relevance of the cross-section difference for the $v_{\mu} \rightarrow v_{\mu}$ disappearance analysis [10]. Confidence regions in the $\left(\theta_{23}, \Delta m_{31}^{2}\right)$ plane obtained when the data simulated within the SF approach are fitted using the migration matrices from the RFG (solid lines) and SF (shaded areas) calculations. Assumed prior uncertainties are $20 \%$ for the shape and for the normalization.

interactions, essential to reproduce the positions of the QE peaks accurately. All these effects are neglected in the RFG model, which treat the nucleus as a fragment of noninteracting nuclear matter of uniform density in a constant potential.

As QE scattering is the dominant mechanism of neutrino interactions at energies $\sim 1 \mathrm{GeV}$, it can be expected to play an important role in the next generation of oscillation studies. In HyperKamiokande experiment, the narrow off-axis beam will be peaked at $0.6 \mathrm{GeV}$ [1]. In Deep Underground Neutrino Experiment (DUNE) [2], the region of the second oscillation maximum-much more sensitive to the Dirac phase than the first one-will correspond to $\sim 0.8 \mathrm{GeV}$.

The authors of Ref. [10] have analyzed how the difference between the RFG model and the SF approach in the QE channel influences the oscillation analysis. They have considered a $v_{\mu} \rightarrow v_{\mu}$ disappearance experiment similar to $\mathrm{T} 2 \mathrm{~K}$, but employing carbon as the target material, assuming 5 years of data taking at the $750-\mathrm{kW}$ beam power. The GLoBES analysis of Ref. [10] has yielded $\sim 650$ ( 730) charged-current QE events in the SF approach (RFG model), together with $\sim 410$ events of the same topology coming from other interaction mechanisms and $\sim 250$ neutral-current background events. The difference between the QE event numbers in these two approaches, resulting from the difference between the corresponding cross sections, has been found to contribute to a non-negligible effect for the oscillation analysis.

The right panel of Fig. 1 shows the 1, 2 and $3 \sigma$ confidence regions in the $\left(\theta_{23}, \Delta m_{31}^{2}\right)$ plane for the true event rates from the SF approach. The shaded areas are obtained for the fitted rates from the same approach, while the solid lines correspond to those from the RFG model. In these results of Ref. [10], one can observe a shift between the true values of the oscillation parameters-represented by the dot—and the extracted best-fit point—-marked by the triangle—at a $\sim 1 \sigma$ confidence level, both in the mixing angle and the squared mass difference.

As in Ref. [10] the difference between the RFG model and the SF approach has been analyzed 
only for the charged-current $\mathrm{QE}$ predictions, the result in the right panel of Fig. 1 should be regarded as a lower limit of the influence of nuclear-model uncertainties, and this analysis should be extended to consistently take into account multinucleon processes [11, 12] and final-state interactions [5]. Moreover, for resonance excitation, similar differences can be expected based on the available theoretical results [13], and also in the deep-inelastic region, the current modeling of nuclear effects has been shown inaccurate by the recent results from the MINERvA experiment [14].

As a final remark, I would like to note that the success of the next-generation of neutrinooscillation experiments is going to require significant reduction of systematic uncertainties. Such a reduction can only be achieved by coordinated theoretical and experimental efforts aiming at improving existing descriptions of nuclear effects, testing them against available lepton-scattering data, implementing in Monte Carlo generators, and performing new cross-section measurements. However, the discoveries coming in the next two decades have the potential to overshadow those of the past two decades.

This work is supported by the National Science Foundation under Grant PHY-1352106.

\section{References}

[1] K. Abe et al. (Hyper-Kamiokande Collaboration), Prog. Theor. Exp. Phys. 2015 (2015) 053C02.

[2] R. Acciarri R. et al. (DUNE Collaboration), Long-Baseline Neutrino Facility (LBNF) and Deep Underground Neutrino Experiment (DUNE). Conceptual Design Report. Volume 2: The Physics Program for DUNE at LBNF, arXiv: 1512.06148.

[3] A. M. Ankowski and C. Mariani, Systematic uncertainties in long-baseline neutrino-oscillation experiments, arXiv:1609.00258.

[4] O. Benhar, D. Day, and I. Sick, Rev. Mod. Phys. 80 (2008) 189.

[5] A. M. Ankowski, O. Benhar, and M. Sakuda, Phys. Rev. D 91 (2015) 033005.

[6] R. A. Smith and E. J. Moniz, Nucl. Phys. B 43 (1972) 605; Erratum: Nucl. Phys. B 101 (1975) 547.

[7] D. T. Baran et al., Phys. Rev. Lett. 61 (1988) 400.

[8] O. Benhar, A. Fabrocini, and S. Fantoni, Nucl. Phys. A 505 (1989) 267.

[9] O. Benhar, A. Fabrocini, S. Fantoni, and I. Sick, Nucl. Phys. A 579 (1994) 493.

[10] C.-M. Jen, A. M. Ankowski, O. Benhar, A. P. Furmanski, L. N. Kalousis, and C. Mariani, Phys. Rev. D 90 (2014) 093004.

[11] O. Benhar, A. Lovato, and N. Rocco, Phys. Rev. C 92 (2015) 024602.

[12] N. Rocco, A. Lovato, and O. Benhar, Phys. Rev. Lett. 116 (2016) 192501.

[13] O. Benhar and D. Meloni, Nucl. Phys. A 789 (2007) 379.

[14] B. G. Tice et al. (MINERvA Collaboration), Phys. Rev. Lett. 112 (2014) 231801. 